\title{
Metapneumovirus humano como causa de hospitalización en niños bajo 3 años de edad, con infección respiratoria aguda, durante el año 2004
}

\author{
M. Alejandra Prado S., Cecilia Perret P., Luisa Montecinos P., Ana Veloz B., Nicole Le Corre P., \\ Leila Habash A., Marcela Potin S., Katia Abarca V. y Marcela Ferrés G.
}

\section{Human metapneumovirus as hospitalization cause in children under 3 years old with acute respiratory infections during 2004}

Human metapneumovirus was detected in 15 of 123 children (12\%) younger than 3 years of age hospitalized for treatment of acute respiratory infection between July and November 2004. The virus was detected by RTPCR directly from nasopharyngeal swabs and/or from supernatants after cell culture. Children infected with hMPV were mostly younger than one year of age (67\%), all presenting with fever and cough. The main cause for hospitalization was the need for oxygen therapy (73\%). Four hMPV positive children had an identifiable co-morbid condition but had a similar clinical evolution when compared to previously healthy infants. Chest radiography showed an increase in interstitial infiltrates with focal consolidation in 6 children. Obstructive bronchial syndrome and bronchiolitis, with or without pneumonia, were the most frequent diagnosis associated with hMPV positivity. A rapid and sensitive diagnostic method is required to improve diagnosis and treatment of these patients.

Key words: Metapneumovirus, respiratory tract infections, reverse transcriptase-polymerase chain reaction (RT-PCR).

Palabras claves: Metapneumovirus, infecciones tracto respiratorio, reacción de polimerasa en cadenatranscripción reversa (RPC-TR).

\section{Introducción}

$\mathrm{L}$ as infecciones respiratorias agudas (IRA) son causa importante de morbimortalidad en niños en todo el mundo ${ }^{1}$. La mayoría de estas IRA son causadas por virus respiratorios, dentro de ellos, el virus respiratorio sincicial (VRS), influenza A y B, parainfluenza 1, 2 y 3 y adenovirus (ADV). Sin embargo, existe un porcentaje no despreciable de IRA, probablemente de etiología viral, en el que no es posible identificar el agente causal, y que representa entre 40 y $60 \%$ de las neumonías adquiridas en la comunidad ${ }^{2-4}$.

En el año 2001, van den Hoogen, en los Países Bajos, descubrió un nuevo virus respiratorio en niños que cursaban con una IRA, y en quienes el estudio etiológico viral tradicional era negativo (VRS, ADV, influenza A y B y parainfluenza 1, 2 y 3) ${ }^{5}$. Este nuevo virus se denominó metapneumovirus humano (MPVh) por su semejanza con el metapneumovirus aviar ${ }^{6}$. Has- ta ahora, se conocen dos linajes o líneas genéticas, A y B, y en cada una de ellas, se han identificado dos subtipos, 1 y $2^{7-10}$.

A la fecha, este agente ha sido descrito en Países Bajos $^{5}$, Inglaterra ${ }^{11}$, Finlandia ${ }^{12}$, España ${ }^{8}$, E.U.A. ${ }^{13}$, Canadá $^{9}{ }^{14,15}$, Brasil ${ }^{16}$, Argentina ${ }^{17}$, México ${ }^{4}$, Uruguay ${ }^{18}$ y recientemente también en Chile ${ }^{19}$ y Perú $^{20}$. Epidemiológicamente tiene una distribución estacional, predominando en invierno ${ }^{21,22}$. Es una infección de alta frecuencia en lactantes y preescolares; estudios de seroprevalencia han mostrado la presencia de anticuerpos anti MPVh en casi $100 \%$ de los niños a la edad de 5 años ${ }^{5}$.

Desde el punto de vista clínico, la bronquiolitis es la manifestación más frecuente en niños bajo 24 meses de edad ed,13,23,24 $^{4}$ pero puede ser responsable de un espectro más amplio de síntomas respiratorios, como por ejemplo infecciones respiratorias superiores y neumonías graves ${ }^{5,13-15,22,25}$. Usualmente la infección es leve a
Pontificia Universidad Católica de Chile y Hospital Clínico Pontificia Universidad Católica de Chile, Santiago. Chile Laboratorio de Infectología y Virología Molecular, Departamento de Pediatría.

Recibido: 2 de marzo 2006 Aceptado: 2 agosto 2006

\section{Correspondencia a:}

M. Alejandra Prado Sanhueza mprado@med.puc.cl 
moderada; sin embargo, se han descrito casos fata$\operatorname{les}^{14,26-30}$, algunos de ellos en pacientes sin patología previa $^{28-30}$.

La presencia de MPVh en nuestro país se ha documentado entre los años 2002 y 2004, dentro del Programa de Vigilancia de Virus Respiratorios de la Pontificia Universidad Católica de Chile y en el trabajo publicado recientemente por Luchsinger ${ }^{19}$. En este sistema de vigilancia, se lo ha encontrado en 17, 3,4 y 9,5\% de los niños bajo 2 años de edad con IRA y panel viral negativo ${ }^{31}$.

El objetivo del presente estudio es estimar la frecuencia de MPVh en pacientes hospitalizados por IRA, bajo 3 años de edad y en quienes no se identificó otra etiología viral respiratoria, así como describir el patrón clínico y epidemiológico de la infección por este virus en Santiago de Chile.

\section{Pacientes y Método}

Estudio prospectivo de niños bajo 3 años de edad, hospitalizados por IRA en el Servicio de Pediatría del Hospital Clínico de la Pontificia Universidad Católica de Chile durante el año 2004, con estudio viral negativo (esto es, detección de antígeno de VRS, ADV, influenza A y B, parainfluenza 1, 2 y 3 negativos por inmunofluorescencia directa (IFD) realizada con Kit Respiratory Virus Panel PathoDx, Lenexa, KS, E.U.A.). No se realizó detección de éstos ni otros agentes virales mediante cultivo viral ni RPC, así como tampoco búsqueda de microorganismos como Bordetella pertussis o Mycoplasma pneumoniae.

Previo consentimiento informado, en cada paciente se obtuvo dos muestras de hisopado nasofaríngeo (HNF) para la determinación de MPVh. En la primera de las muestras se realizó RPC, previa transcripción reversa (RPC-TR) y en la segunda se realizó cultivo viral en células LLC-MK2, para observación de efecto citopático (ECP) y RPC-TR de aquellas muestras con ECP y del resto de las muestras a los 21 días de observación. Para el cultivo viral se utilizaron células LLCMK2 crecidas en medio MEM-Earle's suplementado con albúmina al 1\%, HEPES $1 \mathrm{M}$, glucosa $100 \mathrm{X}$, penicilina-estreptomicina $100 \mathrm{X}$ y tripsina pancreática porcina $0,1 \%{ }^{14,15}$. Se inoculó $200 \mu 1$ de la muestra, previa adsorción de $45 \mathrm{~min}$, se incubó a $37^{\circ} \mathrm{C}$ en atmósfera con $5 \% \mathrm{CO}_{2}$ por 21 días. Las células fueron observadas cada tres días en busca de ECP. El ECP fue definido como el hallazgo de células oscuras granulares separadas de la monocapa.

Se registró en un cuestionario las características clínicas y radiológicas de cada uno de los pacientes. Para homogenizar los diagnósticos de egreso de los pacientes, se definió bronquiolitis como el primer episodio de obstrucción bronquial en un lactante bajo un año de edad, síndrome bronquial obstructivo (SBO) al segundo episodio o siguientes de obstrucción bronquial (presencia de sibilancias, espiración prolongada o roncus), neumonía al compromiso parenquimatoso en la radiografía de tórax, que no correspondía a una atelectasia y compromiso mixto cuando además del componente obstructivo, éste se asociaba a compromiso parenquimatoso.

Las muestras de HNF fueron transportadas a $4^{\circ} \mathrm{C}$ al Laboratorio de Infectología y Virología Molecular de la Universidad Católica de Chile y congeladas de inmediato a $-70^{\circ} \mathrm{C}$ hasta que fueron procesadas.

Técnica de RPC-TR: Para el análisis de RPC-TR, la extracción de ARN se realizó con los reactivos High Pure Nucleic Acid (Roche Diagnostic Molecular Biochemicals, Mannheim, Germany) siguiendo las instrucciones de los fabricantes. Se amplificó una porción del gen de la proteína de fusión (F) de 347 pares de base $^{25}$ y cuando este resultado fue positivo, se confirmó con la amplificación de la nucleoproteína (N) de 213 pares de base de acuerdo a lo publicado por Mackay I y cols ${ }^{32}$. La cepa de MPVh usado como control positivo para RPC-TR y cultivo, fue donada por Guy Boivin del Centre Recherches en Infectiologie, Québec, Canadá.

Los partidores usados para los genes de las proteínas $\mathrm{F}$ y $\mathrm{N}$ fueron MPV-F-R1-5'GAA-AACTGCCGC ACAACATTTAG-3', MPV-F-F1-5' GAGCAAATTGA AAATCCCAG-ACA-3, ${ }^{25}$ y MPV01-2-5, AACCG TGTACTAAGTGATGCACTC-3', MPV02-2-5' CATTGTTTGACCGGCCCCATAA- ${ }^{3}{ }^{32}$, respectivamente.

$\mathrm{El}$ ensayo para el gen de la proteína $\mathrm{F}$ fue realizado en una mezcla que contenía tampón de reacción $10 \mathrm{x}$, 0,2 $\mathrm{mM}$ dNTPs, 0,5 uM de cada partidor, $3 \mathrm{mM}$ de $\mathrm{MgCl}_{2}, 0,1 \mathrm{U} / \mu \mathrm{L}$ de $\mathrm{M}-\mathrm{MLV}$ transcriptasa reversa (Invitrogen), 0,1 U/ $\mu \mathrm{L}$ de Taq $\mathrm{ADN}$ polimerasa (Invitrogen), $3 \mu \mathrm{L}$ de ARN extraído y agua libre de nucleasas hasta completar un volumen de $30 \mu \mathrm{L}$. Se realizó un primer ciclo para la transcripción reversa de 45 minutos a $48^{\circ} \mathrm{C}, 2$ minutos a $94{ }^{\circ} \mathrm{C}$, seguido por 30 ciclos de RPC de un minuto a $94{ }^{\circ} \mathrm{C}, 45$ segundos a $56{ }^{\circ} \mathrm{C}$, 45 segundos a $72{ }^{\circ} \mathrm{C}$ y una extensión final a $72{ }^{\circ} \mathrm{C}$ por 5 minutos. En la etapa de amplificación se utilizó el termociclador Gene Amp PCR System 2400 (Perkin Elmer).

El ensayo para el gen de la proteína $\mathrm{N}$ se realizó en una mezcla que contenía tampón de reacción $10 \mathrm{x}, 0,4$ $\mathrm{mM}$ dNTPs, $0,2 \mu \mathrm{M}$ de cada partidor, $3 \mathrm{mM}$ de $\mathrm{MgCl}_{2}$, $0,1 \mathrm{U} / \mu \mathrm{L}$ de $\mathrm{M}-\mathrm{MLV}$ transcriptasa reversa, $0,1 \mathrm{U} / \mu \mathrm{L}$ de Taq ADN polimerasa, $3 \mu \mathrm{L}$ del ARN extraído y agua libre de nucleasas hasta completar un volumen de 30 $\mu \mathrm{L}$. Las condiciones para la amplificación consistieron 
en 30 minutos a $50{ }^{\circ} \mathrm{C}$, seguido por 30 ciclos de 20 segundos a $94^{\circ} \mathrm{C}, 55^{\circ} \mathrm{C}$ y $68^{\circ} \mathrm{C}$ y una extensión final a $68^{\circ} \mathrm{C}$ por 7 minutos. Los productos de amplificación de RPC se visualizaron en un gel de agarosa. Los fragmentos del tamaño esperado se recuperaron desde el gel y fueron secuenciados en el servicio de secuenciación de la Pontificia Universidad Católica de Chile en el secuenciador automático de ADN ABI3100 (Applied Biosystem) confirmando la amplificación de genoma de MPVh.

Análisis estadístico: Para comparar variables continuas se utilizó test de Mann-Whitney y para variables categóricas test de $\chi^{2}$ o test exacto de Fischer cuando fue necesario, considerando un valor $\mathrm{p}<0,05$ como significativo.

\section{Resultados}

Se ingresó un total de 123 pacientes al estudio. En 74 se realizó RPC-TR de la muestra de HNF, en 20 se realizó sólo cultivo (con RPC-TR del sobrenadante al encontrar ECP o al completar 21 días en cultivo) y en 29 se realizó ambas técnicas.

En 15 casos se logró identificar MPVh. Todos ellos mediante RPC-TR para las proteínas F y N. De los 15 casos positivos, en 6 se detectó el virus por RPC-TR desde sobrenadante del cultivo; en $4 / 6$ se pudo visualizar ECP, en promedio a los 13,5 días de incubación. Los otros 9 casos positivos fueron obtenidos por RPC-TR directamente de la muestra de HNF. En 9 de las 15 muestras positivas se realizaron dos RPC-TR: directa de la muestra y del sobrenadante a los 21 días de cultivo; en 4 de ellas (44\%) hubo concordancia (Tabla 1). Hubo 2 casos en que RPC-TR directa del HNF fueron negativas, pero el resultado del sobrenadante del cultivo fue positivo.

Los casos se detectaron entre la semana 27 y la semana 48 (Figura 1), predominando en los meses de julio y agosto del 2004.

La edad promedio de los niños con MPVh fue de 13,3 meses (rango: 24 días a 34 meses), con una mediana de 11 meses, la que comparada con los niños con estudio negativo no muestra diferencia significativa $(\mathrm{p}>0,05)$. La distribución por género no demostró predominio de ninguno de ellos $(8$ hombres y 7 mujeres) (Tabla 2).

De los 15 pacientes con infección por MPVh, un tercio (5) tenían antecedentes mórbidos de importancia: 2 recién nacidos prematuros, uno de ellos con displasia broncopulmonar y síndrome bronquial obstructivo recurrente (SBOR), un niño con síndrome de Down, uno con SBOR y uno con antecedente de cardiopatía y daño pulmonar crónico.
Los síntomas más frecuentes de los casos positivos al momento de consultar fueron tos, en $100 \%$ de los casos, fiebre en $93 \%$ y dificultad respiratoria en $87 \%$. Otros síntomas menos frecuentes se pueden apreciar en la Tabla 3. El tiempo de evolución previo a la toma de la muestra de hisopado nasofaríngeo fue de 3,9 días (rango: 1-7 días).

El principal motivo de hospitalización fue la necesidad de oxígeno, ya que $73 \%$ tenía saturación menor a $94 \%$ al momento de consultar. Adicionalmente, se pudo objetivar fiebre en $60 \%$ de ellos y al examen físico segmentario se describieron crepitaciones en $80 \%$, faringe congestiva en $60 \%$ y signología obstructiva en $60 \%$.

El tiempo total de hospitalización fue en promedio de 5,6 días (rango: 2-17) y el promedio de uso de oxígeno fue de 3,7 días (rango: 1-7).

Requirieron ingreso a Unidad de Paciente Crítico (UPC) 4 pacientes $(27 \%)$, con una estadía promedio de 1,5 días. Los 4 pacientes fueron trasladados por requerimiento de oxígeno mayor de 30\%; sin embargo, ninguno de ellos requirió ventilación mecánica. Ningún paciente falleció.

El enfrentamiento terapéutico incluyó la administración de oxígeno en $93 \%$, con un máximo de $50 \%$ de $\mathrm{FiO}_{2}$, broncodilatadores en $93 \%$, antimicrobianos en $53 \%$ y corticoesteroides sistémicos en $40 \%$.

\begin{tabular}{|c|c|c|c|}
\hline & $\begin{array}{c}\text { RPC-TR } \\
\text { directa del HNF }\end{array}$ & $\begin{array}{c}\text { RPC-TR a } 21 \text { días } \\
\text { de cultivo }\end{array}$ & $\begin{array}{c}\text { Efecto } \\
\text { citopático }\end{array}$ \\
\hline 1 & + & + & + \\
\hline 2 & - & + & + \\
\hline 3 & + & - & - \\
\hline 4 & + & NR & NR \\
\hline 5 & + & + & - \\
\hline 6 & + & NR & NR \\
\hline 7 & + & + & - \\
\hline 8 & - & + & + \\
\hline 9 & + & NR & NR \\
\hline 10 & + & - & - \\
\hline 11 & + & - & - \\
\hline 12 & + & + & + \\
\hline 13 & + & NR & NR \\
\hline 14 & + & NR & NR \\
\hline 15 & + & NR & NR \\
\hline
\end{tabular}




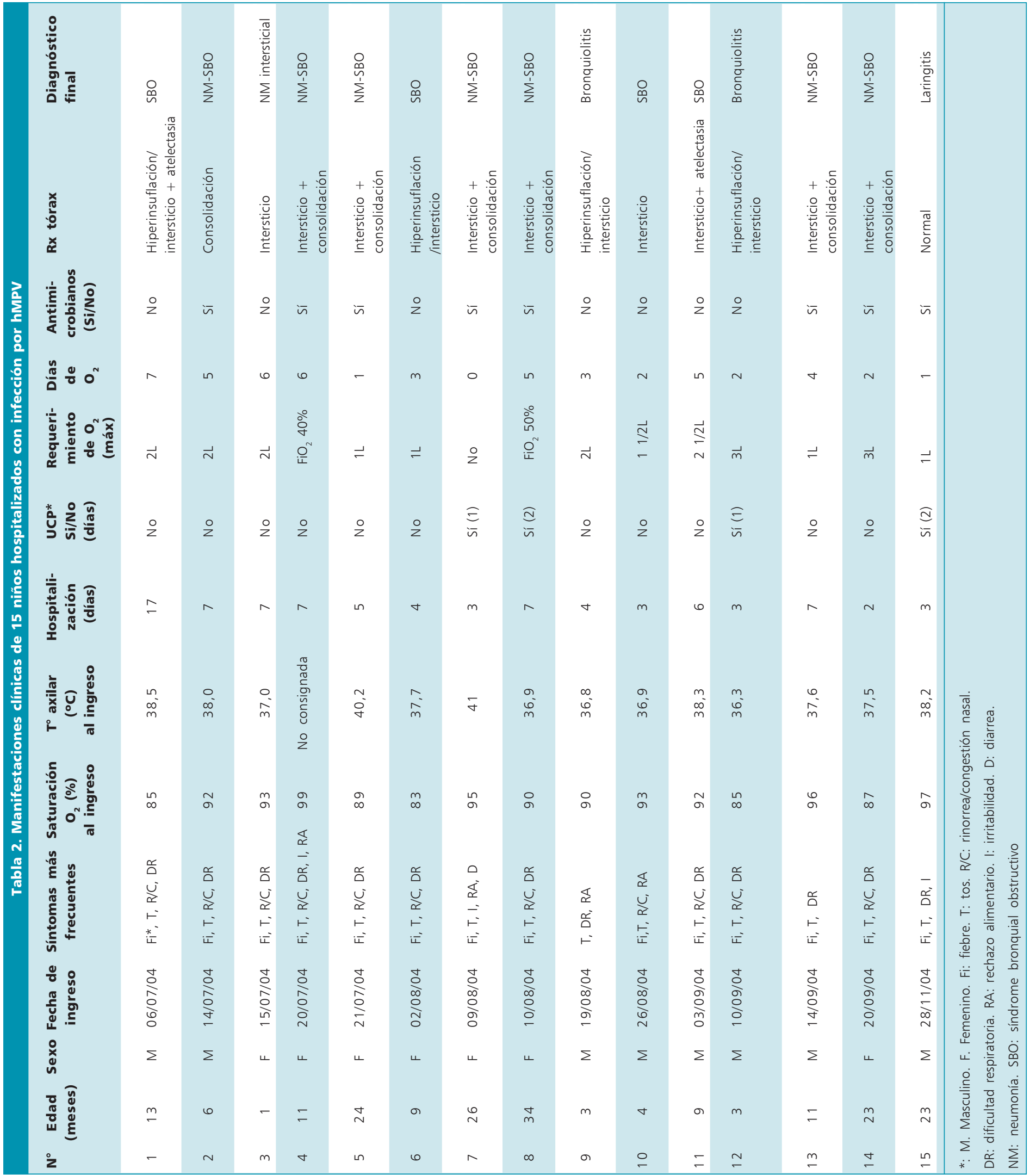




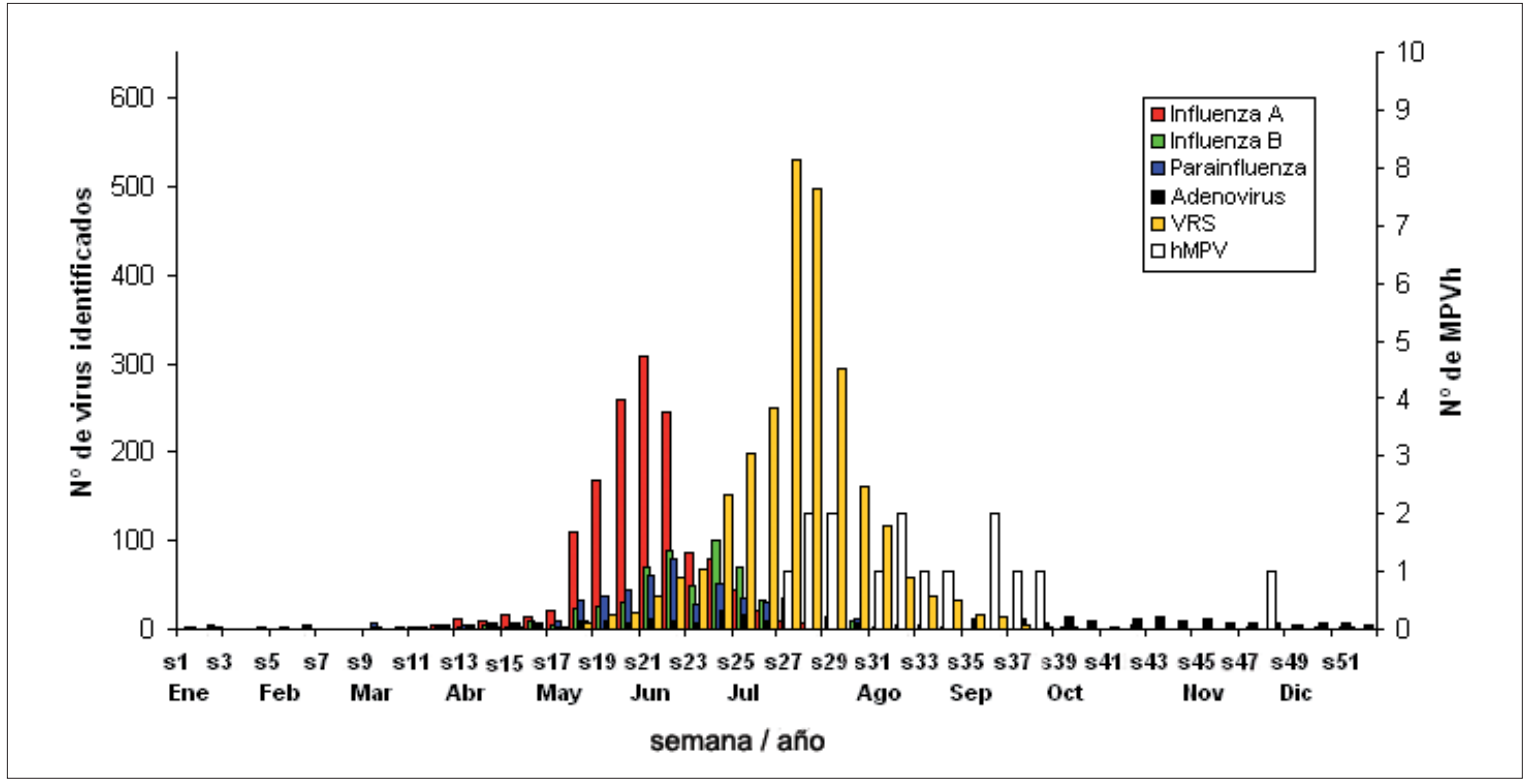

El patrón radiológico predominante fue el compromiso intersticial en 13 pacientes, asociado a hiperinsuflación en 4 de ellos y a consolidación en 6 (Figuras 2 y 3 ).

El espectro de los diagnósticos de egreso fue SBO o bronquiolitis (40\%), síndrome bronquial obstructivoneumonía (SBO-NM) (46\%), neumonía intersticial (7\%) y laringitis (7\%), siendo el más frecuente SBO-NM.

No hubo diferencia estadísticamente significativa en cuanto a estadía hospitalaria, días de oxígeno ni uso de antimicrobianos entre los niños sin antecedentes y aquellos con patología de base.

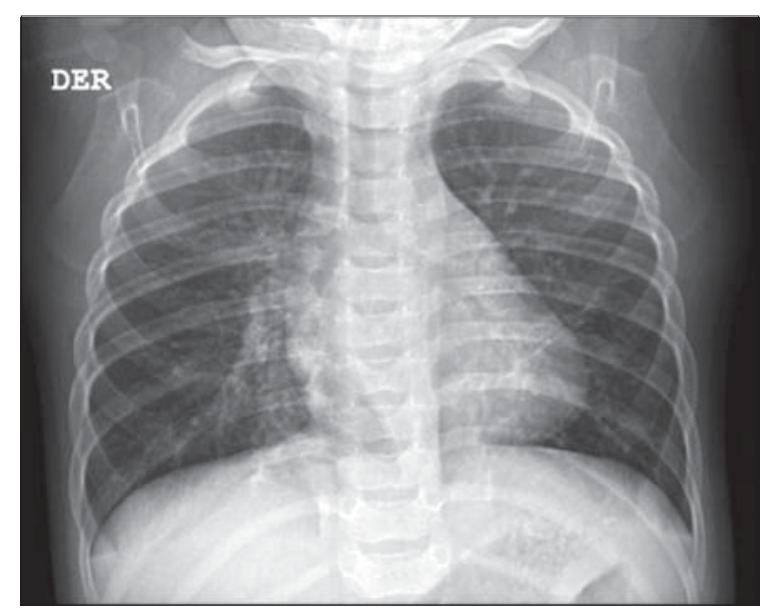

Figura 2. Radiografía de tórax de pacientes con hMPV. Se aprecian hiperinsuflación e imágenes intersticiales.
Tabla 3. Síntomas más frecuentes en los pacientes con MPVh al momento de la primera consulta

\begin{tabular}{lrr} 
Síntoma & $\mathbf{n}$ & $\mathbf{( \% )}$ \\
Tos & 15 & $(100)$ \\
Fiebre & 14 & $(93)$ \\
Dificultad respiratoria & 13 & $(87)$ \\
Rinorrea/congestión nasal & 11 & $(73)$ \\
Rechazo alimentario & 4 & $(27)$ \\
Irritabilidad & 3 & $(20)$ \\
Diarrea & 1 & $(7)$ \\
\hline
\end{tabular}

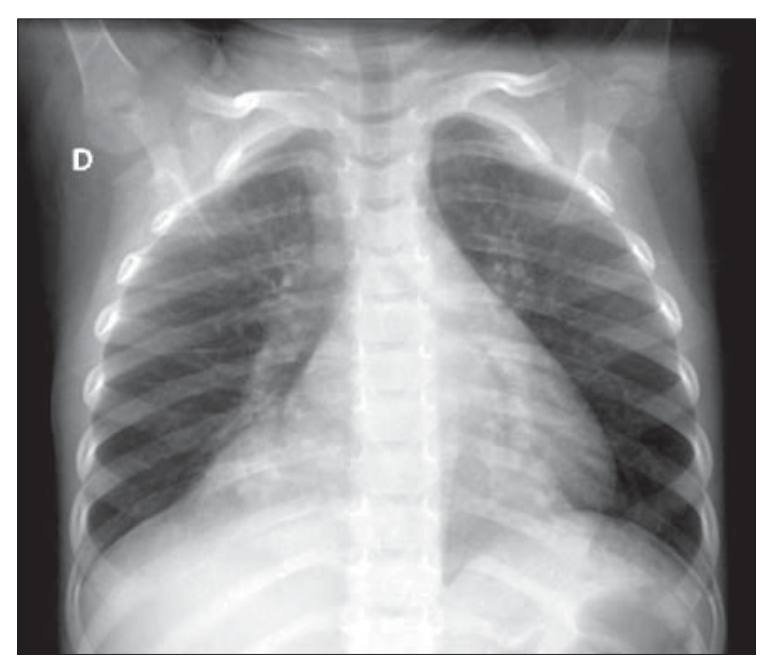

Figura 3. Radiografía de tórax de pacientes con hMPV. Se observa además imagen de consolidación en el lóbulo medio derecho.
Figura 1. Comparación de otros virus respiratorios detectados en niños hospitalizados en el sistema de vigilancia de la PUC con los casos detectados de hMPV en el año 2004 (nótese que hay una escala distinta para hMPV). 


\section{Discusión}

Este estudio describe la presencia de MPVh en $12,2 \%$ de los niños bajo 3 años de edad hospitalizados por cuadros de IRA durante el año 2004, en quienes no se logró identificar ninguno de los virus respiratorios tradicionalmente estudiados. Este hallazgo es concordante con la literatura donde se describe entre 4 y $17 \%$ de infección por MPVh en poblaciones simila$\operatorname{res}^{4,8,16,19,24,33}$.

Durante el año 2004, la detección de MPVh se inició en el período de mayor frecuencia de VRS extendiéndose más allá de la circulación de éste (Figura 1). Ambos virus son causa de sintomatología similar, lo que hace difícil su diferenciación desde el punto de vista clínico ${ }^{5,17,21,22,24,33}$. Actualmente, el diagnóstico de VRS por técnicas de laboratorio es fácilmente accesible en la práctica diaria; sin embargo, hasta la fecha, no existe una amplia difusión y uso de las pruebas de diagnóstico rápidas de MPVh. En esta serie encontramos que $12 \%$ de los niños bajo 3 años de edad con panel viral negativo presentaba infección por hMPV, porcentaje que podría estar subestimado pues no se estudiaron niños con panel viral positivo que pudiesen estar co-infectados con MPVh. La co-infección con VRS está descrita con frecuencias variables entre 0 y $70 \%$, según los grupos estudiados $8,13,14,16,21,24,34,35$. Buscar dicha asociación es un elemento importante a considerar en futuros estudios.

Clínicamente, la mayoría de los pacientes presentaron cuadros respiratorios con predominio de obstrucción bronquial y crepitaciones que hicieron pensar en neumonía. En 7 de ellos se confirmaron los dos diagnósticos: SBO y NM, con el hallazgo de consolidación en la radiografía de tórax. Si bien el compromiso respiratorio bajo fue el más frecuente, se diagnosticó un caso de laringitis ("croup"), situación poco habitual, pero que ha sido descrita previamente ${ }^{4,23,33}$.

Sólo un cuarto de los pacientes con MPVh requirió ingreso a Unidad de Paciente Crítico, sin requerir en ningún caso ventilación mecánica. $\mathrm{Si}$ bien todos los cuadros de esta serie fueron leves a moderados, se ha descrito que MPVh puede tener una evolución más grave, con compromiso rápidamente progresivo e incluso muerte ${ }^{14,26-30}$.

El patrón radiológico predominante fue de hiperinsuflación/patrón intersticial (Figuras 2 y 3), con algunas imágenes de relleno alveolar y/o de atelectasias, lo que concuerda plenamente con lo descrito en publicaciones previas ${ }^{21,33,36}$. El $46 \%$ tuvo compromiso alveolo-intersticial, y en la mayoría de ellos (6 de 7) se usó antimicrobianos por presentar exámenes alterados (hemograma, PCR). Esto enfatiza la necesidad de disponer de un diagnóstico rápido y precoz de este agen- te etiológico para reducir el uso de antimicrobianos. Además, un diagnóstico temprano de MPVh nos permitirá tomar las medidas de aislamiento adecuadas para prevenir su transmisión nosocomial.

Metapneumovirus humano nuevamente muestra un patrón estacional, como se ha podido ver en el sistema de vigilancia de la PUC durante los años 2002, 2003 y $2004^{31}$ (comunicación personal de Dra. Marcela Ferrés). En el presente estudio los primeros casos aparecieron en julio, predominando claramente en los meses de invierno, detectándose casos aislados hasta fines de noviembre, con un patrón más tardío y prolongado que VRS (Figura 1). Este patrón estacional con predominio en invierno se describe en la mayoría de los trabajos publicados ${ }^{21,22}$, pero cada vez más se describe también durante el período de primavera ${ }^{17,19,24,36}$.

Si bien no podemos asegurar que el hallazgo de MPVh en secreción nasofaríngea sea la causa de la infección respiratoria, esto parece lo más probable, ya que los pacientes tenían síntomas de una infección respiratoria, asociado a una radiografía alterada, en la mayoría de los casos, en ausencia de los virus respiratorios clásicos. En este estudio no se buscó demostrar si existe portación asintomática, ya que el diseño incluyó sólo pacientes con síntomas respiratorios; sin embargo, algunos estudios muestran que la portación existe, aunque es poco frecuente f $^{33,37}$.

Respecto de las técnicas diagnósticas, MPVh es difícil de aislar en líneas celulares, por lo que el cultivo no representa una técnica de diagnóstico de primera línea en la práctica clínica ${ }^{5,9,14,20,24,33,38}$. Las técnicas serológicas son útiles, principalmente, para estudios epidemiológicos ${ }^{5,18,25}$. La técnica de RPC-TR representa hoy el método de elección en diagnóstico virológico $9,13,14,21,23-25,32,33,36,38$; no obstante, tanto su excelente sensibilidad como técnica, su costo y la necesidad de una infraestructura especial para su ejecución, hacen necesaria la disponibilidad de métodos de diagnóstico sensibles, específicos y rápidos, como la IF o el ELISA ${ }^{39,40}$ para un uso masivo en el diagnóstico de IRA por MPVh.

Al analizar las concordancias y diferencias encontradas entre las metodologías diagnósticas usadas en paralelo, llama la atención el resultado negativo de dos muestras en la RPC-TR directa desde el HNF y el resultado positivo de ellas en la muestra de sobrenadante del cultivo, en este caso se podría plantear que el ADN se degradó y no pudo ser detectado en el análisis. Entre las causas probables podemos argumentar una muestra con alta contaminación bacteriana, una incorrecta toma de muestra, error en la cadena de frío hasta el laboratorio, no pudiendo precisar cuál fue la causa final de esta observación. En el caso contrario, en que el resultado de RPC-TR de la muestra direc- 
ta de HNF fue positivo y el resultado del sobrenadante fue negativo, sabemos que MPVh es un virus difícil de cultivar y que la sensibilidad de esta técnica es mucho menor que la de RPC-TR ${ }^{20,33}$.

Metapneumovirus humano representa hoy un nuevo agente causal de infecciones respiratorias que debe ser incluido en el diagnóstico diferencial de las IRA, pudiendo afectar tanto a niños sanos como a algunos con patología crónica. Clínicamente, se presenta en la mayoría de los pacientes con tos, fiebre y dificultad respiratoria, indistinguible de otros virus respiratorios, como VRS, siendo el requerimiento de oxígeno la causa más frecuente de hospitalización. El patrón radiológico más característico es el compromiso intersticial, que puede estar o no asociado a compromiso alveolar. El diagnóstico se realiza actualmente por medio de RPC-TR.

\section{Resumen}

Metapneumovirus humano (MPVh) fue detectado entre julio y noviembre en 15 de 123 niños bajo 3 años de edad hospitalizados por infección respiratoria aguda $(12 \%)$. Las muestras fueron estudiadas mediante técnicas de biología molecular (RPC-TR de muestra de hisopado nasofaríngeo y/o de sobrenadante de cultivo). El $67 \%$ de los niños hospitalizados con MPVh tenían menos de 1 año de edad, todos ellos presentaron tos y fiebre y el principal motivo de hospitalización fue el requerimiento de oxígeno en $73 \%$ de los casos. Si bien un tercio de los pacientes tenía patología previa, su evolución clínica no fue diferente respecto de los niños previamente sanos. El patrón radiológico mostró aumento de la trama intersticial, con focos de consolidación en 6 casos (40\%). El diagnóstico más frecuente fue síndrome bronquial obstructivo o bronquiolitis, asociado o no a neumonía. Destaca la necesidad de un método de diagnóstico rápido para optimizar el diagnóstico diferencial, manejo y control de infecciones en estos pacientes.

\section{Agradecimientos}

En forma especial a Guy Boivin del Centre Recherches en Infectiologie, Québec, Canadá, por la donación de la cepa viral usada como control positivo. Al personal de enfermería del Hospital Clínico de la Pontificia Universidad Católica de Chile. A Paola Viviani, por el análisis estadístico.

\section{Referencias}

1.- Bryce J, Boschin Pinto C, Shibuya K, Black R E and the WHO Child Health Epidemiology Reference Group. WHO estimates of the causes of death in children. Lancet North Am Ed 2005; 365: 1147-52.

2.- Davies H D, Matlow A, Petric M, Glazier R, Wang E. Prospective comparative study of viral, bacterial and atypical organism identified in pneumonia and bronchiolitis in hospitalized Canadian infants. Pediatr Infect Dis J 1996; 15: 371-5.

3.- Ruiz M, Ewig S, Marcos M A, Martínez J A, Arancibia F, Mensa J, et al. Etiology of community-acquired pneumonia: impact of age, comorbidity, and severity. Am J Respir Crit Care Med 1999; 169: 397-405.

4.- Noyola D E, Alpuche-Solis AG, HerreraDíaz A, Soria-Guerra R E, SánchezAlvarado J, López-Revilla R. Human metapneumovirus infections in Mexico: epidemiological and clinical characteristics. J Med Microbiol 2005; 54: 969-74.

5.- van den Hoogen B G, de Jong J C, Groen J, Kuiken T, de Groot R, Fouchier R A, et al. A newly discovered human pneumovirus isolated from young children with respiratory tract disease. Nat Med 2001; 7: 719-24.
6.- van den Hoogen B G, Bestebroer T M, Osterhaus A D, Fouchier R A. Analysis of the genomic sequence of a human metapneumovirus. Virology 2002; 295: 119-32.

7. - van den Hoogen B, Herfst S, Sprong L, Cane P A, Forleo-Neto E, de Swart R L, et al. Antigenic and genetic variability of human metapneumoviruses. Emerg Infect Dis 2004; 10: 658-66.

8.- Vicente D, Cilla G, Montes M, PérezTrallero E. Human metapneumovirus and community-acquired respiratory illness in children. Emerg Infect Dis 2003; 9: 602-3.

9.- Peret T, Boivin G, Li Y, Couillard M, Humphrey C, Osterhaus A, et al. Characterization of human metapneumovirus isolated from patients in North America. J Infect Dis 2002; 185: 1660-3.

10.- Biacchesi S. Skiadopoulos M H, Boivin G, Hanson C T, Murphy B R, Collins P L, et al. Genetic diversity between human metapneumovirus subgroups. Virology 2003; 315: $1-9$.

11.- Stockton J, Stephenson I, Fleming D, Zambon M. Human metapneumovirus as a cause of community-acquired respiratory illness. Emerg Infect Dis 2002; 8: 897-901.

12.- Jartti T, van den Hoogen B, Garofalo R P, Osterhaus A D, Ruuskanen O.
Metapneumovirus and acute wheezing in children. Lancet 2002; 360: 1393-4.

13.- Esper F, Boucher D, Weibel C, Martinello R A, Kahn J S. Human metapneumovirus infection in the United States: Clinical manifestations associated with a newly emerging respiratory infection in children. Pediatrics 2003; 111: 1407-10.

14.- Boivin G, Abed Y, Pelletier G, Ruel L, Moisan D, Cote S, et al. Virological features and clinical manifestations associated with human metapneumovirus: A new paramyxovirus responsible for acute respiratory-tract infections in all age groups. J Infect Dis 2002; 186: 1330-4.

15.- Bastien N, Ward D, van Caeseele P, Brant K, Lee S, McNabb G, et al. Human metapneumovirus infection in the Canadian population. J Clin Microbiol 2003; 41: 4642-6.

16.- Cuevas L, Ben Nasser A, Dove W, Gurgel R, Greensill J, Hart A. Human metapneumovirus and respiratory syncytial virus, Brazil. Emerg Infect Dis 2003; 9: 1626-8.

17. - Galiano M, Videla C, Puch S S, Martínez A, Echavarria M, Carballal G. Evidence of human metapneumovirus in children in Argentina. J Med Virol 2004; 72: 299-303. 
18.- Mirazo S, Ruchansky, Blanc A, Arbiza J. Serologic evidence of human metapneumovirus circulation in Uruguay. Mem Inst Oswaldo Cruz, Río de Janeiro 2005; 100: 715-8.

19.- Luchsinger V, Escobar C, Avendaño L. Detection of human metapneumovirus in children hospitalized for acute lower children respiratory infection in Santiago, Chile. Rev Méd Chile 2005; 133: 1059-64.

20.- Gray G C, Capuano A W, Setterquist S F, Sánchez J L, Neville J S, Olson J, et al. Human metapneumovirus, Peru. Emerg Infect Dis 2006; 12: 347-50.

21.- van den Hoogen B G, van Doornum G J, Fockens J C, Cornelissen J J, Beber W E, de Groot R, et al. Prevalence and clinical symptoms of human metapneumovirus infection in hospitalized patients. J Infect Dis 2003; 188: 1571-7.

22.- van den Hoogen B G, Osterhaus D, Fouchier R A. Clinical impact and diagnosis of human metapneumovirus infection. Pediatr Infect Dis J 2004; 23: S25-32.

23.- Freymuth F, Vabret A, Legrand L, Eterradossi N, Lafay-Delaire F, Brouard J, et al. Presence of the new human metapneumovirus in French children with bronchiolitis. Pediatr Infect Dis J 2003; 22: 92-4.

24.- Boivin G, De Serres G, Cote S, Gilca R, Abed Y, Rochette L, et al. Human metapneumovirus infections in hospitalized children. Emerg Infect Dis 2003; 9: 634-40.

25.- Falsey A R, Erdman D, Anderson L, Walsh E. Human metapneumovirus infections in young and elderly adults. J Infect Dis 2003; 187: 785-90.

26.- Pelletier G, Déry P, Abed Y, Boivin G. Respiratory tract reinfections by the new human metapneumovirus in an immunocompromised child. Emerg Infect Dis 2002; 8: 976-8.

27.- Cane P A, van den Hoogen B G, Chakrabarti S, Fegan C D, Osterhaus A D. Human metapneumovirus in a haematopoietic stem cell transplant recipient with fatal lower respiratory tract disease. Bone Marrow Transplant 2003; 31: 309-10.

28.- Chan P K S, To K F, Wu A, Tse G M K, Chan K F, Lui S F, et al. Human metapneumovirus-associated atypical pneumonia and SARS. Emerg Infect Dis 2004; 10: 497-500.

29.- Schildgen O, Glatzel T, Geikowski T, Scheibner B, Matz B, Bindl L, et al. Human metapneumovirus RNA in encephalitis patient. Emerg Infect Dis 2005; 11: 467-70.

30.- Le Corre N, Montecinos L, Prado P, Donoso A, Drago M, Wegner A, Ferres M. Detección de metapneumovirus humano (hMPV) en cuadros respiratorios rápidamente progresivos con desenlace fatal en 3 centros hospitalarios de Santiago, 20042005. Libro de resúmenes XXII Congreso Chileno de Infectología. 26-29 octubre 2005, Puerto Varas. Resumen CO 32, pág 71.

31.- Ferrés M, Depix M, Azócar T, Contreras I, Ovalle J, Veloz A, et al. Metapneumovirus humano en infección respiratoria aguda en menores de 2 años. Libro de resúmenes XX Congreso Chileno de Infectología. 5-8 noviembre 2003, La Serena. Resumen CO 13, pág 42.

32.- Mackay I, Jacob K, Woolhouse D, Walker K, Syrmis M W, Whiley D M, et al. Molecular assays for detection of human metapneumovirus. J Clin Microbiol 2003; 41:100-5

33.- Williams J V, Harris P A, Tollefson S J,
Halburnt-Rush L L, Pingsterhaus J M, Edwards K M, et al. Human metapneumovirus and lower respiratory tract disease in otherwise healthy infants and children. N Engl J Med 2004; 350: 443-50.

34.- Greesill J, McNamara P S, Dove W, Flanagan B, Smyth R L, Hart C A. Human metapneumovirus in severe respiratory syncytial virus bronchiolitis. Emerg Infect Dis 2003; 9: 372-5.

35. - Lazar I, Weibel C, Dziura J, Ferguson D, Landry M L, Kahn J S. Human metapneumovirus and severity of respiratory syncytial virus disease. Emerg Infect Dis 2004; 10: 1318-20.

36.- Esper F, Martinello R A, Boucher D, Weibel C, Ferguson D, Landry ML, et al. A 1-year experience with human metapneumovirus in children aged $<5$ years. J Infect Dis 2004; 189: $1388-96$

37.- Osterhaus A, Fouchier R. Human metapneumovirus in the community. Lancet 2003; 361: 890-1.

38.- Chan P K S, Tam J S, Lam Ch-W, Chan E, $\mathrm{Wu} \mathrm{A}$, Li Ch-K, et al. Human metapneumovirus detection in patients with severe acute respiratory syndrome. Emerg Infect Dis 2003; 9: 1058-63.

39. - Landry M L, Ferguson D, Cohen S, Peret TCT, Erdman DD. Detection of human metapneumovirus in clinical samples by immunofluoresecnce staining of shell vial centrifugation cultures prepared from three different cell lines. J Clin Microbiol 2005; 43: 1950-2.

40.- Percivalle E, Sarasini A, Visai L, Revello G, Gerna G. Rapid detection of human metapneumovirus strains in nasopharyngeal aspirates and shell vial cultures by monoclonal antibodies. J Clin Microbiol 2005; 43: 3443-6. 